\title{
Correction to: Deoxynivalenol-induced alterations in the redox status of HepG2 cells: identification of lipid hydroperoxides, the role of Nrf2-Keap1 signaling, and protective effects of zinc
}

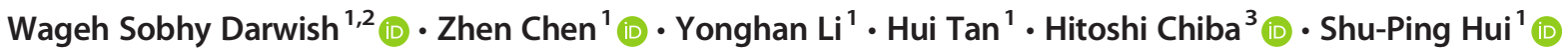 \\ Published online: 23 April 2020 \\ (C) Society for Mycotoxin (Research Gesellschaft für Mykotoxinforschung e.V.) and Springer-Verlag GmbH Germany, part of Springer Nature 2020
}

\section{Correction to: Mycotoxin Research} https://doi.org/10.1007/s12550-020-00392-x

- The original version of this paper was published with error: Wageh Sobhy Darwish, Zhen Chen, Yonghan Li, Tan Hui, Hitoshi Chiba and Shu-Ping Hui. Authors apologize for making an error in the presentation of the name of the author Tan Hui, it should be Hui Tan.

- The corrected author presentation is Wageh Sobhy Darwish, Zhen Chen, Yonghan Li, Hui Tan, Hitoshi Chiba and Shu-Ping Hui.
- This mix between family name and first name of Hui Tan happened during a final editing of the manuscript and passed unnoticed during submission and revision. We like to apologize for this mistake and thank Mycotoxin Research Editor-in-Chief and journal manager for giving us the chance to correct this error.

Publisher's note Springer Nature remains neutral with regard to jurisdictional claims in published maps and institutional affiliations.
The online version of the original article can be found at https://doi.org/ $10.1007 /$ s $12550-020-00392-\mathrm{x}$

Shu-Ping Hui

keino@hs.hokudai.ac.jp

1 Laboratory for Advanced Lipid Analysis, Faculty of Health Sciences, Hokkaido University, Kita 12, Nishi 5, Sapporo, Japan

2 Food Control Department, Faculty of Veterinary Medicine, Zagazig University, Zagazig 44519, Egypt

3 Department of Nutrition, Sapporo University of Health Sciences, Nakanuma Nishi 4-2-1-15, Higashi Ku, Sapporo 007-0894, Japan 